\title{
COMPETENCIAS DEL DOCENTE CLÍNICO ENFERMERA/O, UNIVERSIDADES LLEIDA (ESPAÑA) Y LA FRONTERA (CHILE): PERCEPCIÓN DEL ESTUDIANTE
}

\section{COMPETENCES OF CLINICAL TEACHER NURSES, UNIVERSIDAD DE LLEIDA (SPAIN) AND THE LA FRONTERA (CHILE): STUDENT PERCEPTION}

\author{
Mónica Illesca Pretty \\ Mirtha Cabezas González ${ }^{* *}$ \\ Carmen Nuin OrRio** \\ PiLAR JÜRSCHIK JiMÉNEZ ${ }^{* * * *}$
}

\begin{abstract}
RESUMEN
El objetivo es comparar las opiniones de estudiantes de Enfermería de las universidades de Lleida y de La Frontera (UFRO) respecto a la percepción de las competencias que debe tener una enfermera/o tutor/a para la docencia clínica. Investigación cualitativa, mediante Técnica de Redes Semánticas Naturales. Población constituida por todos los estudiantes de segundo y tercer año (110 Lleida y 63 UFRO), a quienes en forma individual -previo Consentimiento Informado- se les solicitó escribieran en cinco minutos diez palabras (verbos, adverbios, adjetivos, sustantivos) relacionadas a la pregunta estímulo: “Cuáles son las características que debe tener una Enfermera/o para la docencia clínica?”, priorizándolas posteriormente de uno a diez, correspondiendo el uno al de mayor valor. El análisis se realizó mediante reducción progresiva de la información de acuerdo a la técnica mencionada. Estudiantes de ambas escuelas coinciden que el atributo "empatía" (100\%) es la principal actitud esperable para enfermeras/os que realizan docencia clínica. En Lleida "docente" es mencionada en un $80,1 \%$ por segundo año y $43,1 \%$ por tercero; mientras que en UFRO, en un $47,2 \%$ los de segundo y $23 \%$ tercero. La "experiencia", en Lleida, corresponde al $86,3 \%$ para segundo y $42 \%$ para tercero, palabra no mencionada por UFRO. El "conocimiento" aparece para segundo año-UFRO en un 89,8\% y 47,1\% en tercero, en Lleida sólo lo identifican los de segundo en un $36,4 \%$. Las competencias genéricas es el rasgo fundamental en el quehacer docente clínico, según los estudiantes de ambas escuelas. La característica "docente" se puede atribuir a competencias genéricas y específicas.
\end{abstract}

Palabras clave: Competencia clínica, docente de enfermería, educación en enfermería, actitud.

\begin{abstract}
The objective is to compare the views of nursing students at the University of Lleida and the University of La Frontera (UFRO), in relation to the perception of the skills that should have a nurse or tutor in the clinical teaching. Qualitative research, the Techniques of Natural Semantic Nets was used. Sample. All students from second and third year (110 Lleida and 63 UFRO) participated, whom prior signed Informed Consent, were asked, individually and in five minutes, write ten words (verbs, adverbs, adjectives, nouns) related to the

\footnotetext{
* Enfermera. Académica Medicina Interna. Universidad de La Frontera. Temuco, Chile. E-mail: millesca@ufro.cl

** Enfermera. Académica Ciencias Preclínicas. Universidad de La Frontera. Temuco, Chile. E-mail: mcabezas@ufro.cl

${ }^{* * *}$ Máster en Ciencias de la Enfermería. Profesora Titular Escuela de Enfermería, Universidad de Lleida. Lleida, España.

E-mail: carmen.nuin@infermeria.udl.cat

${ }^{* * * *}$ Doctora en Medicina. Profesora Escuela de Enfermería, Universidad de Lleida. Lleida, España. E-mail: pilar.jurschik@ infermeria.udel.cat
} 
stimulus question "What are the characteristics that should have a nurse for clinical teaching". Thereafter, they should rank the words, corresponding the number one to the higher value. Analysis through reduction of the information according to the technique. All students from both schools agree that "empathy" $(100 \%)$ is the main attitude expected from nurses who perform clinical teaching. In Lleida, "teaching" is mentioned in second and third year with $80.1 \% 43.1 \%$, respectively, in UFRO represents $47.2 \%$ second year and $23 \%$ third year. The "experience", in Lleida, is $86.3 \%$ for second year and $42 \%$ for third year, attributes not mentioned by the UFRO'students. For the second year (UFRO) "knowledge" $89.8 \%$ and $47.1 \%$ third year, this aspect is identified only for the second year of Lleida with a $36.4 \%$. For the students from both schools the generic competence are the fundamental attributes for the clinical teaching. The characteristic "teaching" may be considered to the competence of to be, knowledge and know-how.

Key words: Clinical competence, nursing teacher, nursing education, attitude.

Fecha recepción: 11/06/09 Fecha aceptación: 06/05/10

\section{INTRODUCCIÓN}

En la formación de los profesionales enfermera/o, tanto en España como en Chile, las experiencias clínicas constituyen uno de los principales quehaceres para la adquisición de las competencias disciplinares y genéricas para la gestión del cuidado (1). Durante esta práctica, los estudiantes son monitorizados por profesionales de la respectiva disciplina, quienes son los responsables de facilitar el proceso enseñanza-aprendizaje (2), y no necesariamente tienen la formación en esta área. Además, el desafío de propiciar el desarrollo de estas capacidades actitudinales y profesionales es mayor, ya que el ambiente de aprendizaje generado en la realidad de un centro de salud es totalmente diferente al que existe en el aula, el cual es diseñado para el cumplimiento de objetivos determinados.

Como resultado de un trabajo conjunto entre las Escuelas de Enfermería de la Universidad de Lleida, España, y la Universidad de La Frontera, UFRO, Chile, desde el año 2004 a la fecha se ha observado similitud en los planes de estudio, en lo que se refiere a la adquisición de competencias genéricas y disciplinares, las que se logran, en lo fundamental, en el ambiente clínico-asistencial. Sin embargo existe una diferencia, los estudiantes de Lleida realizan la práctica en servicios de salud supervisados por profesiona- les enfermera/os asistenciales cuyo contrato laboral es prioritariamente del centro de salud, denominados profesores asociados, mientras que los de la UFRO son monitorizados por enfermeras/os contratados por la institución.

Dicha diferencia permitió a los docentes de ambas instituciones reflexionar sobre la percepción que tendrían los estudiantes en cuanto a las competencias que demuestran los profesores en la práctica docente-clínica. Esto da origen al supuesto de si la condición contractual afecta en las competencias de los profesionales que forman estudiantes en los dos centros asistenciales. Por tanto, se planteó la interrogante el año 2007 si existen diferencias en la opinión de los educandos de segundo y tercer año en dichas escuelas, en cuanto a las competencias que tienen las enfermeras/os que supervisan las prácticas clínicas.

Si bien es cierto existen variadas definiciones de cuidado, como por ejemplo atender las necesidades emocionales, sociales y físicas del usuario, asistir en la realización de las actividades que contribuyen a la salud o a su recuperación para obtener independencia a la mayor brevedad posible $(3,4)$, la reflexión de Leininger (5), quien los clasifica en genéricos y profesionales, se compatibiliza con el desarrollo de competencias disciplinares y genéricas que deben adquirir los educandos, ajustándose a la inquietud de los autores. Ella 
sostiene que son saberes que deben adquirirse en las universidades para finalmente capacitar a individuos y comunidades donde las técnicas y procesos de cuidado se orientan al mantenimiento y desarrollo de condiciones favorables de vida y de muerte.

Las competencias genéricas corresponden al trabajo en equipo, análisis crítico de la información, liderazgo, tomas de decisiones, respeto, entre otras (6-8) y las profesionales a todas aquellas propias de la disciplina para lograr las competencias requeridas en las funciones asistencia, investigación, docencia y gestión $(9,10,11)$.

En un ambiente de aprendizaje complejo y multifuncional (12) los estudiantes deben desempeñar distintas acciones tendientes a la gestión del cuidado mediante la interacción constante entre el usuario, el estudiante, enfermera/o asistencial (Servicio de Salud) y enfermera/o de la institución educativa, la que no puede ser reemplazada por otra didáctica $(13,14,15)$. En un tiempo determinado asumen activamente el rol, se enfrentan a un contexto socio-cultural específico, en el cual debe primar una reflexión permanente de sus acciones (16). Sumado a lo anterior, están las particularidades derivadas de la mediación de objetivos de aprendizaje con el docente clínico, el cumplimiento de protocolos para la función asistencial, lo concerniente a lo administrativo y necesidades educativas tanto del usuario y familia como del equipo de enfermería.

Es evidente que en este ambiente de aprendizaje se genera un alto nivel de estrés tanto para el educando (17) como para el docente, siendo estos últimos quienes deben demostrar capacidades en los dominios del saber aprender: autorregulación de la propia práctica y al trabajo conjunto con otros académicos; dominio del saber: investigación para la producción de conocimiento, teorías y metodologías educativas, así como la cultura organizacional de la institución; dominio del saber hacer: destrezas didácticas para facilitar al educando el desarrollo de habilidades para otorgar cuidados; dominio del ser: óptimas relaciones interpersonales con sus pares y estudiantes, de modo de constituirse como modelos de formación $(18,19)$.

Para estos efectos se establece una investigación cualitativa, con enfoque interpretativo, basada en la perspectiva hermenéutica y émica, es decir comprender el complejo mundo de experiencia vivida desde el punto de vista de las personas que la viven, en este caso del informante $(20,21,22)$. La recolección y análisis de la información se llevó a cabo mediante la técnica de Redes Semánticas Naturales (23). La decisión de optar por este método se basa en la naturaleza del objeto de estudio: reconstruir las formas de sentir, pensar y actuar de los estudiantes con respecto al tema a investigar $(24,25)$, quienes, siendo los actores más importantes del proceso enseñanza-aprendizaje, darán luces a los responsables de los planes de estudio para proponer mejoras en la calidad de la docencia clínica $(26,27,28,29)$.

Además el estudio permitirá la construcción de conocimientos y la internalización de las competencias genéricas en docentes y futuros profesionales, aspectos que se han vuelto primordiales tanto en las instituciones de educación superior como en lo laboral.

\section{MATERIAL Y MÉTODO}

Investigación cualitativa, mediante Técnica de Redes Semánticas Naturales (23), cuyo objeto de estudio son discentes de Enfermería, de las universidades de Lleida y de La Frontera, quienes a partir de sus historias personales y colectivas proporcionaron información válida con relación a las características que debe tener la enfermera/o que realiza supervisión clínica.

La población en estudio, intencionada, se conformó con todos los estudiantes de Lleida y UFRO: segundo año $(65$ y 33$)$ y tercer año (45 y 30 ), respectivamente. El criterio de in- 
clusión fue pertenecer durante el 2007 a los niveles mencionados y disponer de tiempo para participar en la investigación.

Después de firmar el Consentimiento Informado (25) se aplicó un instrumento diseñado para recolectar la información y su análisis posterior, según Técnica de Redes Semánticas (23).

Dicho procedimiento consistió en solicitar a los sujetos participantes que definieran, individualmente, en cinco minutos como límite de tiempo, con diez palabras (verbos, adverbios, adjetivos, sustantivos o pronombres, sin utilizar artículos o preposiciones) la pregunta estímulo o reactivo: "cuáles son las características que debe tener una enfermera/o para la supervisión clínica”. A la vez, se les pidió que asignaran a cada palabra definidora un número del uno al diez, para luego jerarquizarlas, de acuerdo a la importancia atribuida (uno la más importante, diez la menos importante).

Consecuente con la técnica, para el análisis se procedió a la reducción de datos mediante sinónimos y merónimos para obtener los cuatro indicadores establecidos: Valor J (total de las palabras definidoras); Valor M (multiplicando frecuencia de aparición, FA, por el valor semántico, VS, para cada palabra definidora, convirtiendo las jerarquías asignadas por los sujetos, jerarquía 1 vale 10 puntos, la 2 vale 9 puntos y así sucesivamente; Conjunto SAM (doce palabras definidoras con mayor valor M) y Valor FMG (obtenido mediante una proporción, correspondiendo al $100 \%$ la palabra definidora con el valor $\mathrm{M}$ más grande) (23).

La validación se llevó a cabo mediante la triangulación por investigadores y expertos $(30,31)$. Finalmente se elaboró un set de información definitivo que reflejó la coincidencia de los significados de todos los autores que participaron en el estudio.

Se declaró el compromiso por parte de los investigadores a mantener la confidencialidad de la información, excepto para fines de difusión en eventos científicos. Es importante destacar que los sujetos investigados tuvieron libertad de elección para participar mediante la firma del Consentimiento Informado.

\section{RESULTADOS}

Las palabras totales definidoras de los estudiantes del segundo año de la Escuela de Lleida fueron 190 y en la UFRO 97, por sistema de sinonimia y meronimia, según triangulación del estudio, fueron reducidas a 43 (Lleida) y 52 (UFRO). Para el tercer año de Lleida fueron 108 y siguiendo el esquema de reducción quedaron 59; mientras que en la UFRO las 66 palabras definidoras se redujeron a 38 (Tabla 1).

Tabla 1. Reducción palabras definidoras segundo y tercer año Universidad de Lleida y Universidad de La Frontera - 2007.

\begin{tabular}{|c|c|c|c|c|c|c|}
\cline { 2 - 7 } \multicolumn{1}{c|}{} & \multicolumn{2}{c|}{ LLEIDA } & \multicolumn{3}{c|}{ UFRO } \\
\cline { 2 - 7 } \multicolumn{1}{c|}{} & Estudiantes & $\begin{array}{c}\text { Total palabras } \\
\text { definidoras }\end{array}$ & $\begin{array}{c}\text { Sinónimos y } \\
\text { merónimos }\end{array}$ & Estudiantes & $\begin{array}{c}\text { Total palabras } \\
\text { definidoras }\end{array}$ & $\begin{array}{c}\text { Sinónimos y } \\
\text { merónimos }\end{array}$ \\
\hline Segundo año & 65 & 190 & 43 & 33 & 97 & 52 \\
\hline Tercer año & 45 & 108 & 66 & 30 & 59 & 38 \\
\hline
\end{tabular}


En la Tabla 2 se muestra que la palabra "amable" tiene una frecuencia de 100\%, para el grupo del segundo año de Lleida, en UFRO lo es el atributo "empática" (100\%) para el mismo nivel curricular de los estudiantes. La "experiencia" en Lleida es de un $86,3 \%$ y "conocimiento" para UFRO es de 89,8\%. La condición "docente" para Lleida es de 80,1\% y $47,2 \%$ para la UFRO. Las otras características se refieren a competencias genéricas con excepción de "conocimiento" 36,4\% (Lleida) e “inteligente" 27\% (UFRO).
Los estudiantes del tercer año, para ambas instituciones (Tabla 3), definen el atributo "empático" en un 100\%. La condición "docente" para los estudiantes de Lleida corresponde a un 43,1\%, mientras que para los de la UFRO es de un 23\%. La "experiencia” para los de Lleida corresponde a un $42 \%$, no siendo mencionado por los estudiantes UFRO. El "conocimiento" para Lleida no es identificado pero sí para los alumnos de la UFRO con un $47,1 \%$. El resto de los términos del Conjunto SAM conciernen a competencias genéricas.

Tabla 2. Comparación estudiantes de segundo año Universidad de Lleida y Universidad de La Frontera - 2007.

\begin{tabular}{|l|c|c|l|c|c|}
\hline \multicolumn{2}{|c|}{ Segundo año Lleida (65 estudiantes) } & \multicolumn{2}{c|}{ Segundo año UFRO (33 estudiantes) } \\
\hline $\begin{array}{c}\text { CONJUNTO } \\
\text { SAM }\end{array}$ & VALOR M & $\begin{array}{c}\text { VALOR } \\
\text { FMG }\end{array}$ & $\begin{array}{c}\text { CONJUNTO } \\
\text { SAM }\end{array}$ & VALOR M & $\begin{array}{c}\text { VALOR } \\
\text { FMG }\end{array}$ \\
\hline Amable & 321 & $100 \%$ & Empática & 197 & $100 \%$ \\
\hline Experiencia & 277 & $86.3 \%$ & Conocimiento & 177 & $89.8 \%$ \\
\hline Paciencia & 258 & $80.4 \%$ & Tolerante & 104 & $52.8 \%$ \\
\hline Docente & 257 & $80.1 \%$ & Docente & 93 & $47.2 \%$ \\
\hline Simpática & 195 & $60.7 \%$ & Respetuosa & 76 & $38.6 \%$ \\
\hline Empática & 184 & $57.3 \%$ & Comprensiva & 67 & $34.0 \%$ \\
\hline Comprensiva & 176 & $54.8 \%$ & Responsable & 60 & $30.4 \%$ \\
\hline Responsable & 126 & $39.3 \%$ & Amigable & 55 & $27.9 \%$ \\
\hline Conocimiento & 117 & $36.4 \%$ & Inteligente & 53 & $26.9 \%$ \\
\hline Comunicación & 111 & $34.6 \%$ & Sincera & 46 & $23.3 \%$ \\
\hline Humano & 104 & $32.4 \%$ & Confiable & 42 & $21.3 \%$ \\
\hline Respetuosa & 101 & $31.5 \%$ & Solidaria & 38 & $19.3 \%$ \\
\hline
\end{tabular}

Tabla 3. Comparación estudiantes de tercer año Universidad de Lleida y Universidad de La Frontera - 2007.

\begin{tabular}{|l|c|c|l|c|l|}
\hline \multicolumn{2}{|c|}{ Tercer año Lleida (45 estudiantes) } & \multicolumn{2}{c|}{ Tercer año UFRO (30 estudiantes) } \\
\hline $\begin{array}{c}\text { CONJUNTO } \\
\text { SAM }\end{array}$ & VALOR M & $\begin{array}{c}\text { VALOR } \\
\text { FMG }\end{array}$ & $\begin{array}{c}\text { CONJUNTO } \\
\text { SAM }\end{array}$ & VALOR M & $\begin{array}{c}\text { VALOR } \\
\text { FMG }\end{array}$ \\
\hline Empática & 276 & $100 \%$ & Empática & 244 & $100 \%$ \\
\hline Amable & 209 & $75.7 \%$ & Respetuosa & 129 & $52.9 \%$ \\
\hline Disponible & 206 & $74.6 \%$ & Conocimiento & 115 & $47.1 \%$ \\
\hline
\end{tabular}




\begin{tabular}{|l|c|c|l|c|l|}
\hline Docente & 119 & $43.1 \%$ & Tolerante & 111 & $45.5 \%$ \\
\hline Experiencia & 116 & $42.0 \%$ & Buen Evaluador & 102 & $41.8 \%$ \\
\hline Simpática & 112 & $40.5 \%$ & Confiable & 91 & $37.3 \%$ \\
\hline Organizada & 111 & $40.2 \%$ & Consecuente & 90 & $36.9 \%$ \\
\hline Responsable & 105 & $38.0 \%$ & Líder & 76 & $31.1 \%$ \\
\hline Paciencia & 99 & $35.9 \%$ & Incentivadora & 74 & $30.3 \%$ \\
\hline Comprensiva & 97 & $35.1 \%$ & Objetiva & 63 & $25.8 \%$ \\
\hline Trabajadora & 93 & $33.7 \%$ & Docente & 56 & $23.0 \%$ \\
\hline Respetuosa & 92 & $33.3 \%$ & Autocrítica & 46 & $18.9 \%$ \\
\hline
\end{tabular}

\section{DISCUSIÓN Y COMENTARIOS}

Los resultados difieren a lo esperado por los investigadores, ya que se suponía que por las diferentes condiciones contractuales de los enfermeros/as de ambas instituciones, los estudiantes de Lleida identificarían el "conocimiento" como requerimiento primordial, debido a que son más clínicos y los de la UFRO señalarían la "experiencia", porque tienen un mayor trabajo en aula. Esto se puede interpretar en dos sentidos: ¿están expresando la realidad de la supervisión clínica o están manifestando las características que debería tener la enfermera/o en esta función?

Llama la atención que el atributo "docente" para el total de los participantes ocupa un lugar relevante, interpretándose como el cumplimiento de las competencias del saber, saber hacer y del saber ser.

Además, se destaca que los 173 estudiantes de la investigación señalan que las características más significativas del docente clínico se refieren a las competencias genéricas, concordando con las expectativas de los empleadores a nivel mundial, que para una atención de calidad no se debe soslayar el "humanismo" en toda acción en salud.

Entendiendo que la motivación y satisfac- ción de los estudiantes son factores sensibles para el logro de un aprendizaje significativo, se colige que ambas escuelas deberían diseñar estrategias en la formación de enfermeras/os valorando la relación humana entre educando y profesor/a, conjuntamente al conocimiento disciplinar.

La relevancia de realizar este estudio para los profesionales de la salud que trabajan en un ambiente complejo en los servicios asistenciales y que deben formar estudiantes en las competencias del ser, saber y saber hacer, radica en tener información concreta desde la perspectiva de los educandos, con el fin de utilizar estrategias metodológicas diferentes en la supervisión de las experiencias clínicas, ya que también se compromete en ésta la salud mental de todos los actores involucrados en el proceso educativo, incluyendo al usuario y su entorno familiar.

Cabe destacar en este estudio la participación de todos los estudiantes. Ellos son la pieza clave de cualquier programa efectivo de evaluación de la calidad educativa, pues aportan información valiosa y utilizable. Los datos proporcionados permiten inferir características importantes en la toma de decisiones del funcionamiento de la institución de la que ellos mismos forman parte. 


\section{REFERENCIAS}

1. Serrano P, Martínez M. La tutorización de prácticas clínicas en pregrado de enfermería. Metas Enfermería 2008; 11 (3):28-32.

2. Sanjuan A, Martínez JR. Nuevo enfoque en el proceso de enseñanza-aprendizaje para la interrelación de conocimientos y formación de clínica/comunitaria. Invest Educ Enfermer. 2008; 26 (2 supl): 150159.

3. Swanson EA, Jensen D, Specht J, Jonson M, Maas M. Caregiving: concept analysis and outcomes. Scholarly Inquiry for Nursing Practice: An International Journal 1997; 11 (1): 65-72.

4. Henderson V. Principios Básicos de los Cuidados de Enfermería. 1 $1^{a}$ ed. Suiza: Consejo Internacional de Enfermeras; 1971.

5. Leininger M. Culture care diversity and universality: a theory of nursing. $2^{\mathrm{a}}$ ed. New York: National League for Nursing Press; 1991.

6. Delors J. La educación encierra un tesoro. Informe a la UNESCO de la Comisión Internacional sobre la Educación para el Siglo XXI. $1^{\text {a }}$ ed. Barcelona: España: Grupo Santillana, UNESCO; 1996. pp. 95-108.

7. Tuning Educational Structures in Europe. Informe Final Proyecto Piloto-Fase 1. $1^{\text {a }}$ ed. Universidad de Deusto-Universidad de Groningen. España: Educación y Cultura. Sócrates. 2003.

8. Altieri I E, Hidalgo C, Ibarra P, Moraga A, Soto L. Propuesta Política de Formación General Universidad de La Frontera. Temuco: Universidad de La Frontera. 2007.

9. Falcó A. La nueva formación de profesionales: sobre la competencia profesional y la competencia del estudiante de enfermería. Educación Médica 2004; 7 (1): 42-45.

10. Criterios de Evaluación para la Acredita- ción de Carreras de Enfermería. Hallado en: www.colegiodeenfermeras.cl/info. asp. [Acceso el 14 de mayo de 2009].

11. Libro Blanco de la ANECA para Enfermería. Agencia Nacional de Evaluación de la Calidad y la Acreditación, 2005. Hallado en: http://www.aneca.es/modal_eval/ docs/libroblanco_jun05_enfermeria.pdf [Acceso el 14 mayo de 2009].

12. Medina JL. La Pedagogía del Cuidado: saberes y prácticas en la formación universitaria de Enfermería. $1^{a}$ ed. Barcelona: Alertes; 1999: 191-251.

13. Cid P, Sanhueza O. Acreditación de campos clínicos de enfermería. Rev Cubana Enfermer [revista en la Internet]. 2006 22(4). Hallado en: http://scielo.sld.cu/ scielo. [Acceso el 14 mayo de 2009].

14. Pérez S, Morán L. Percepción de una población de estudiantes de enfermería sobre las características del docente clínico. Desarrollo Científico de Enfermería 2004; 12 (8): 232-236.

15. Parsell G, Bligh J. Recent perspectivas on clinical teaching. Medical Education 2001; 35 (4): 409-414.

16. Schön D. La Formación de profesionales reflexivos. Hacia un nuevo diseño de la enseñanza y el aprendizaje en las profesiones. 1a ed. Barcelona: Paidós; 1992: 17-33.

17. Rodríguez A, Puialto MJ, Moure ML, Quinteiro A. Situaciones de las prácticas clínicas que provocan estrés en los estudiantes de enfermería. Enfermería Global 2007; (10): 1-12.

18. Scriven M. Selección de Profesorado. En Millman J. y Darling-Hammond L. Manual para la evaluación del profesorado. Madrid: La Muralla; 1997: 105-146.

19. Jiménez B. Evaluación de la docencia. En Jiménez, B. Evaluación de programas, centros y profesores. Madrid: Síntesis Educación; 1999: 173-206.

20. Sandín MP. Investigación cualitativa en educación. Fundamentos y tradiciones. $1^{a}$ ed. Madrid: McGraw-Hill/Interameri- 
cana de España; 2003: 119-201.

21. Rodríguez G, Gil J, García E. Metodología de la investigación cualitativa. $1^{\mathrm{a}} \mathrm{ed}$. Málaga: Aljibe; 1996.

22. Pérez G. Investigación cualitativa. Retos e interrogantes. I Métodos Cualitativos. 2a ed. Madrid: La Muralla; 1998.

23. Valdez JL. Las redes semánticas naturales usos y aplicaciones en psicología social. $2^{a}$ ed. México: Universidad Autónoma del Estado de México; 1998: 65-78.

24. Pérez G. Investigación cualitativa. Retos e Interrogantes. II Técnicas y análisis de datos. 2a ed. Madrid: La Muralla; 1998: 101-157.

25. Burns N, Grove SK. Investigación en Enfermería. $3^{a}$ ed. Barcelona: Elsevier; 2004: 172-208.

26. Illesca M, Cabezas M. Satisfacción de los estudiantes en relación con la docencia y administración carrera de enfermería Universidad de la Frontera. Educación en Ciencias de la Salud. 2006; 3 (2): 82-88.

27. Caetano A. Un modelo de gestión para las administraciones públicas. En: VII
Congreso Internacional del CLAD sobre la Reforma del Estado y de la Administración Pública, Lisboa, Portugal, 8-11 Oct. 2002.

28. Ruano C. Más allá de la evaluación por resultados: planteamientos/metodológicos en torno al proceso de autoanálisis institucional y la construcción de indicadores de la calidad educativa en el contexto Universitario 2002. Hallado en http:// www.rieoei.org/deloslectores/367Ruano. PDF [Acceso el 14 mayo de 2009].

29. Santos MA. Criterios de referencia sobre la calidad del proceso de enseñanza/ aprendizaje en la Universidad. En: Primeras Jornadas Nacionales de Didáctica Universitaria. Madrid: Consejo de Universidades; 1990: 49-70.

30. Latorre A, del Rincón D, Arnal J. Bases metodológicas de la investigación educativa. $1^{\text {a }}$ ed. Barcelona: Hurtado; 1996:4950.

31. Stake R. Investigación con estudio de casos. 2a ed. Madrid: Morata; 1999: 94103. 